\title{
A fast and efficient model for real-time anti-tumor drug efficacy test in the clinical setting based on patient-derived tumor xenograft
}

Yanping Zhu ( $\sim$ zhuyanping@personaloncology.cn )

Nanjing Personal Oncology Biotechnology Co.Ltd

Hui Zheng

Nanjing Personal Oncology Biotechnology Co.Ltd

Qian Wang

Nanjing Personal Oncology Biotechnology Co.Ltd

Zhiqiang Wang

Nanjing Personal Oncology Biotechnology Co.Ltd

Hang Song

Nanjing Personal Oncology Biotechnology Co.Ltd

Peng Yuan

National Cancer Center, Peking Union Medical College, Cancer Hospital of Chinese Academy of Medical

Sciences

Deliang Fu

Huashan Hospital『Pancreatic Disease Institute『Fudan University

Yizhou Wang

Shanghai Oriental hepatobiliary surgery hospital

\section{Article}

Keywords: patient-derived tumor xenograft, cancer therapy, clinical setting, engraftment rate

Posted Date: May 17th, 2021

DOl: https://doi.org/10.21203/rs.3.rs-453208/v1

License: (c) (i) This work is licensed under a Creative Commons Attribution 4.0 International License.

Read Full License 


\section{A fast and efficient model for real-time anti-tumor drug efficacy test in the clinical setting}

\section{2 based on patient-derived tumor xenograft}

3 Authors: Hui Zheng ${ }^{1, \# \text {, Qian Wang }}{ }^{1, \#}$, Zhiqiang Wang ${ }^{1}$, Hang Song ${ }^{1}$, Peng Yuan ${ }^{2}$, Deliang $\mathrm{Fu}^{3}$,

4 Yizhou Wang ${ }^{4}$, Yanping Zhu ${ }^{1, *}$

\section{Affiliations:}

$6 \quad \quad{ }^{1}$ Nanjing Personal Oncology Biotechnology Co. Ltd, Nanjing, Jiangsu, China

$7 \quad{ }^{2}$ National Cancer Center, Peking Union Medical College, Cancer Hospital of Chinese Academy

8 of Medical Sciences, Beijing, China

$9 \quad{ }^{3}$ Huashan Hospital, Pancreatic Disease Institute, Fudan University, Shanghai, China

$10 \quad{ }^{4}$ Shanghai Eastern Hepatobiliary Surgery Hospital, Shanghai, China

$11{ }^{*}$ To whom correspondence should be addressed: Dr. Yanping Zhu, Nanjing Personal Oncology

12 Biotechnology Co. Ltd., 2289 East TianYuan Road, Nanjing, 210000, Jiangshu, China.

13 zhuyanping@ personaloncology.cn

14 \# These authors contribute equally

15 The authors declare no potential conflicts of interest. 


\section{Abstract}

21 Drug response rates in cancer therapies are frequently unsatisfactory (first-line 30-40\%,

22 subsequent-line $\sim 10 \%$ or less). The mouse model, patient-derived tumor xenograft (PDTX), can

23 help promote drug effective rate to over $80 \%$ by precisely electing efficacious agents. However,

24 the low engraftment rates, extended testing cycle, and high cost limit its utilization in the clinical

25 setting. A novel method, FastPDTX, was developed, in which a substitutive engrafting procedure

26 was applied, and the labor-intensive and time-consuming propagation of tumorgrafts was

27 circumvented by pathological analysis. A comprehensive evaluation involving tumor biology and

28 clinical performance was performed for this new model. The clinical evaluation engaged 431

29 PDTX cases, 1050 FastPDTX cases, 531 therapeutic entities, 6535 regimens, and a broad spectrum

30 of solid tumors. Typical cases were also studied, covering various refractory malignant tumors.

31 FastPDTX kept biological architectures and genetic characteristics of the primary tumor and

32 displayed an equivalent positive predictive value as PDTX for the drug efficacy test. The testing

33 cycle was reduced from 3-6 months to 3 weeks, and the cost was decreased by $85 \%$. More

34 importantly, the obstacle of low engraftment rate was circumvented, resulting in the percentage of

35 appliable tests nearly 5-fold higher (94.8\% vs. 16.7\%) than PDTX. FastPDTX is particularly

36 suitable in circumstances where standard care is not available and the therapeutic window is short,

37 and for cases where tumors have indolent growth in mice. FastPDTX preserved the advantages of

38 classical PDTX, overcame its major limitations, and showed great potential for real-time clinic

39 drug selection in cancer therapy.

40 Keywords: patient-derived tumor xenograft, cancer therapy, clinical setting, engraftment rate 


\section{$41 \quad$ Background}

42 Immune checkpoint inhibitors (ICIs), the current primary class for cancer immunotherapy,

43 improved clinical outcomes significantly during the past decade. However, just a small subset

$44(\sim 10 \%)$ of patients could benefit from ICIs $(1,2)$, and ICIs as monotherapy have mixed results

45 with modest efficacy if without further stratification(3), and generally, the response rate falls

46 within $20 \%$ to $40 \%$ (4). To improve the efficacy of ICIs, combination with other therapeutic agents

47 becomes the new direction(5). The rationale for combination therapy is turning the immune non-

48 responsive tumor into responsive. Cytotoxic agents can induce cancer cell death, promote tumor

49 antigen release, and stimulate immune effectors. Consequently, an efficacious chemotherapeutic

50 drug will be a natural selection for the combination partner.

51 On the other hand, surgery, followed by adjuvant chemotherapy, targeted therapy, and

52 radiotherapy, remains the mainstay for managing many solid tumors. Nevertheless, the patient

53 response is generally unsatisfactory for current chemotherapy or targeted therapy, and particularly,

54 the response rate usually is less than $10 \%$ after the front-line therapy(6). Furthermore,

55 recommended agents for many rare tumors are usually very limited from guidelines and are not

56 improved for many years (7-10); and personalized medicine is strongly demanded in those

57 circumstances. Therefore, a robust drug screening system that can quickly identify efficacious

58 drugs will significantly impact either ICIs combination therapy and standard cancer care $(11,12)$.

59 Patient-derived tumor xenograft (PDTX) is a type of animal model, which recapitulates various

60 clinical features and captures the therapeutic outcomes effectively, established by directly

61 implanting tumor tissues into an immunodeficient mouse. Applications of PDTX include

62 evaluations of preclinical drugs, basic cancer research, identifications for the biomarkers, and

63 assessments of drug efficacy for personalized therapy(13-19). Particularly, the effective rate of 
64 anti-tumor drugs can be improved to over $80 \%$ under the guidance of $\operatorname{PDTX}(6,16)$; therefore, it

65 is a promising solution for precise drug selection in cancer therapies $(14,16,20-22)$. However,

66 there are still several obstacles for PDTX regarding the utilization for clinical decision making(23),

67 with two essential issues that need to be solved. One difficulty is the low successful engraftment

68 rate (usually around 50\%, varies depending on multiple factors), which substantially reduces the

69 test's applicability. The other challenge is generally a 3-6 months testing cycle that will make the

70 test unsuitable for many cancer patients, especially those at an advanced stage. Other issues include

71 high cost and the requirement of complex animal facilities.

72 In this study, we developed a new modeling protocol, named FastPDTX, that overcomes major

73 disadvantages in PDTX and is more applicable in the clinical setting. In the new FastPDTX

74 protocol, tumorgrafts are implanted in the gluteal region instead of subcutaneously or

75 orthotopically because the enriched blood supply in this region helps a rapid vascularization, and

76 therefore, facilitates drug delivery and the vital status of tumorgrafts. The fundamental alteration

77 is that repetitive measurements of tumorgrafts during the efficacy test are replaced by pathological

78 analysis, which is crucial for reducing time and overcoming the engraftment barrier. We

79 determined key pathological indices and verified their validity through over 200 clinical cases.

80 Our results show that FastPDTX features an equivalent positive predictive value as PDTX for

81 efficacy test while significantly decreases the testing duration by $90 \%$ (from 3-6 months to $2-3$

82 weeks) and the expenses by $85 \%$. More importantly, the barrier of engraftment rates is

83 circumvented, resulting in over $90 \%$ of applicable tests. FastPDTX is particularly suitable for the

84 advanced malignant tumors where standard care is not available and the therapeutic window is

85 limited, and for cases where tumors have indolent growth in mice, which is another obstacle in the

86 traditional PDTX. 
88 Cases and sample sources

891481 patient-derived tumor xenograft cases from four registered multi-center clinical studies

90 (ChiCTR-OOC-17012731, ChiCTR-OOC-17013617, ChiCTR-POC-17013612, ChiCTR-

91 1800018422) and researcher-initiated studies in China covering various cancers were enrolled,

92 including gastric cancer $(N=190)$, colorectal cancer $(N=146)$, liver cancer $(N=497)$, pancreatic

93 cancer $(N=127)$, sarcomas $(N=158)$, gynecological cancer $(N=97)$, cancer of the biliary duct

$94(N=98)$, lung cancer $(N=33)$, breast cancer $(N=25)$, and others malignant tumors (110 rare type

95 cancers). The primary tumoral specimens from surgery, biopsy, or endoscopic aspiration were

96 used as the source for the either PDTX $(N=431)$ or FastPDTX $(N=1050)$ modeling. All protocols

97 followed the guidelines of local institutional ethics regulations and were approved by the local

98 institutional review board. Informed consent was obtained from all patients enrolled.

\section{Animal cares}

100 All recipients for xenograft were 6-8-week-old specific-pathogen-free (SPF)

101 immunocompromised NCG mice (NOD-Prk ${ }^{\mathrm{dcem} 26 \mathrm{Cd} 52}$ Il2rgem26Cd22, Nanjing Biomedical Research

102 Institute, China) weighing $20 \pm 2 \mathrm{~g}$. The research was approved by the Institutional Animal Care

103 and Use Committee (IACUC) of Nanjing Medical University following the National Institutes of

104 Health guide for the care and use of Laboratory animals. All mice were housed in SPF animal

105 facilities at a room temperature of $25 \pm 2^{\circ} \mathrm{C}$ and humidity of $40 \%-70 \%$. Prior to the

106 transplantation, all mice were housed for at least three days for acclimation. The sex of each

107 selected mouse was same as the corresponding tumor donor.

108 Pretreatment of tumor specimen for PDTX or FastPDTX 
109 Freshly collected tumor tissues were immediately preserved in the tissue preservation solution

110 (Hyclone, Marlborough, U.S.A.) with the a supplemental of 100units/ml penicillin and $100 \mathrm{ug} / \mathrm{ml}$

111 streptomycin and were transported to the laboratory under refrigeration within 24 hours. The

112 necrotic components, fats, and blood clots were removed before transplant.

\section{PDTX modeling}

114 The PDTX model was established as described (16). Briefly, 2 X 2X 2mm fresh tumor tissues

115 were engrafted into NCG mice subcutaneously. Tumor volume was measured twice per week and

116 was calculated according to the following formula: tumor volume $=\left[\right.$ length $*$ width $\left.^{2}\right] / 2$. When

117 the volume reached $800-1000 \mathrm{~mm}^{3}$, the initial xenograft (passage 0, P0) was aseptically resected,

118 and was re-partitioned and re-expanded in a similar way for subsequent generations. For the drug

119 test, when the average tumor volume reached $100-150 \mathrm{~mm}^{3}$, the mice were randomly assigned (5

120 mice per group) to control or treatment groups after the substandard mice (weight over

121 Mean \pm SEM/3, SEM, standard error of the mean) removed. The drug interventions were performed

122 for 21 days in a similar manner to the clinical scheme and clinically relevant dose. Tumor volumes

123 of control $(\mathrm{Vc})$ and treatment $(\mathrm{Vt})$ groups were measured to calculate tumor growth inhibition rate

124 (TGI) using the following formula: TGI $=(\mathrm{Vc}-\mathrm{Vt}) / \mathrm{Vc} * 100 \%$. A regimen with $\mathrm{TGI} \geqslant 80 \%$ is

125 designated as effective regimen (positive result).

\section{FastPDTX modeling}

127 The major engrafting procedures were similar as the PDTX modeling with some optimization as 128 described(24). The key alteration in the FastPDTX was tumor tissues were engrafted into the 129 gluteal region where the blood supply is abundant. After the vascularization was established (5 130 days after engrafting), the mice were exposed to candidate agents recommended by oncologists 
131 for 7 days. Drug efficacy was assessed by histopathology and immunohistochemistry analysis of

132 tumor cell necrosis rate (TCNR), and proliferative rate indexed by Ki-67. In addition, the

133 percentage of tumor cells in the tumorgraft was also calculated to further improve accuracy. In the

134 FastPDTX modeling, a therapeutic entity to be considered effective (positive result), it should meet

135 the follow criteria: i.) containing at least 5\% tumor cells in the specimen; ii.) at least one of indices,

136 TCNR or Ki67, in both control and testing samples should be > 5\%; iii.) the TCNR of

137 experiment/control should $>10 \%$ or the ratio of Ki67 of experiment/control should $<80 \%$. The

138 one mouse per model per treatment scheme were adopted since the reproducibility of this scheme

139 has been demonstrated.(25-27) For each tumor, at least 4 unique therapeutic entities were screened

140 for both PDTX and FastPDTX. The schematic paradigm for PDTX and FastPDTX modeling were

141 illustrated in Figure 1A.

\section{Tumor growth rate calculation}

143 Specific growth rate (SGR) was used to quantify tumor growth rate for all tumors by measuring

144 tumor volume changes. The primary tumor tissue was implanted into the same mouse by PDTX 145 and FastPDTX at the same starting time (t1) and with the same starting size (V1), and removed at 146 the same time (t2) when the tumor sizes (V2) were measured. SGR was calculated according to 147 the exponential growth equation as follows: $\mathrm{SGR}=[\ln (\mathrm{V} 2-\mathrm{V} 1)] /(\mathrm{t} 2-\mathrm{t} 1)$.

\section{Positive predictive value and concordant rate}

149 Based on the classification of the response evaluation criteria in solid tumors (RECIST ver1.1),

150 we designated the results as positive clinical responses if the patients' response were within one 151 of the following categories: Complete Response (CR), Partial Response (PR), or Stable Disease 152 (SD). The positive predictive value (PPV) was defined as the percentage of positive clinical 153 responses in PDTX or FastPDTX predicted drug effective cases, i.e., PPV= (number of clinical 
154 positive response cases) / (number of PDTX or FastPDTX predicted positive cases). For the

155 comparison between FastPDTX and classical PDTX, the concordant rate was used. When the

156 predictive result for a drug regimen was same by FastPDTX and PDTX, i.e., both models predicted

157 as effective or noneffective, the case was documented as a concordant case. The concordant rate

$158=$ (number of concordant cases) / (total regimens tested by both FastPDTX and PDTX).

\section{Whole Exome Sequencing (WES) analysis}

160 Genomic DNA extraction and sequencing library construction were performed using standard

161 protocols as described (28). The publicly available software FastQC was used to assess sequencing

162 quality. For each lane, per-base quality scores across the length of the reads were examined. Lanes

163 were deemed passing if the per-base quality score box plot indicated that $>75 \%$ of the reads had >

164 Q20 for bases 1-80. In addition to the raw sequence quality, the alignment quality was also

165 assessed using the Picard package. Whole-exome sequencing was performed on NovaSeq 6000

166 System and the primary base call files were converted into FASTQ sequence files using the

167 bcl2fastq converter tool bcl2fastq-1.8.4 in the CASAVA 1.8 pipeline. The FASTQ sequence files

168 generated were then processed through an in-house pipeline constructed for whole-exome

169 sequence analyses of paired cancer genomes. The sequencing reads were aligned to the reference

170 genome build hg19, GRCh37 using BWA-mem v.0.7.5a(29) and converted into BAM files using

171 SAMtools (version 0.1.18) (30). Duplicates were marked for filtering and INDELs were realigned

172 using GATK v.3.4.46 IndelRealigner (31). For somatic SNV and indel variant calling, GATK

173 BQSR was applied to recalibrate base qualities. SNV and indel somatic variants were called using

174 Strelka v.1.0.14 (32). Copy-number aberration was quantified and reported for each gene as the

175 segmented normalized $\log 2$-transformed exon coverage ratios between each tumor sample and

176 normal samples. Z score was used to measure the significance of copy number variations. For each 
177 specific gene, $\mathrm{Z}$ score $=($ copy number of that gene in the tumorgraft - average copy number of

178 that gene in original tumors)/(standard deviation of copy number of that gene in original tumors).

$179 \mathrm{Z}$ score $>3$ indicates significant copy number variations.

180 Results

181 Optimized tumor growth in FastPDTX

182 In contrast to PDTX, where efficacy testing usually started at stage P3-P5 (tumorgraft passage 3-

183 passege5), and the agent exposure was 21 days, the efficacy assessing in FastPDTX started directly

184 at stage P0 and the during was seven days. The typical drug testing period of FastPDTX was only

185 15-20 days compared to 80-200 days in PDTX (Figure 1A). One precondition for successful drug

186 testing is the well-established blood supply to the tumorgraft for effective drug delivery. Therefore,

187 in the FastPDTX protocol, tumorgrafts were implanted in the gluteal region, where the blood

188 supply is abundant, and presumptively vascularization could be established rapidly. To test this

189 conjecture, a side-by-side comparison was performed, which was conducted by engrafting two

190 same-sized tumor tissues into the same mice using the FastPDTX for one and the PDTX protocol

191 for the other, and after a period of growth, the dimensions of the two tumorgrafts were compared

192 at the same time. The test results clearly demonstrated a more rapid growth of tumorgraft when

193 using the FastPDTX protocol (Figure 1B left). The tumorgraft from the FastPDTX is pinker overall

194 than that from PDTX, indicating a better blood supply inside the graft (Figure 1B right). Clear

195 blood vascular connections were observed from mouse tissue to the graft and inside the graft five

196 days after the implantation (Figure 1C left). An immunohistochemistry inspection was performed

197 using CD31 antibody to verify the establishment of blood supply inside the tumorgraft, and the

198 results also showed a clear vascularization in the graft (Figure 1C right). In the interest of

199 discriminating the difference between two protocols quantically, we further assessed the specific 
200 growth rate (SGR) of tumorgrafts from various types of tumors under both modeling conditions.

201 For all types of tumors, the general SGR under the FastPDTX condition was about 3-9 times higher

202 than PDTX, indicating improved growth environments under the FastPDTX modeling conditions

203 (Figure 1D).

204 FastPDTX preserves the characteristics of original tumors

205 Due to the significant internal differences between human beings and mice, transplanted tumors

206 may alter to adapt to those environmental changes. These alterations may cause discrepancies

207 between the modeling results and the patient's actual response. Therefore, we examined the

208 resemblance between primary tumors and the xenografts from FastPDTX. We did not assess

209 tumorgrafts from the traditional PDTX model since their identity to the primary tumors has already

210 been shown.(16) The first comparison was performed at the histopathologic level. A single-blind

211 method was employed by comparing the concordance between the pathological diagnostic results

212 of primary tumors (Pa) and the paired xenografts (P0). In total, 499 pairs of samples from different

213 types of tumors were examined, and the overall concordance between $\mathrm{Pa}$ and P0 is $98 \%$. Typical

214 images from seven pairs of various tumors are shown (Figure 2A).

215 Copy number variations (CNVs) have been frequently observed in various tumors and are

216 considered influencing drug resistance $(33,34)$. We also examined CNVs for major cancer driver

217 genes in those seven pairs of samples using Whole Exome Sequencing (WES). Only sporadic

218 CNVs between Pa and P0 were discovered, which indicated only minor differences between

219 primary tumors and xenografts regarding the significant DNA fragment changes (Figure 2B).

220 Single nucleotide variation (SNV) or point mutation is the other major contributor to the alterations

221 in drug pharmacokinetics (35). For those seven pairs of samples, we compared nonsynonymous

222 SNVs as well, and a high degree of concordance between Pa and P0 was also observed (Figure 
223 2C). Along with the rarity of CNVs, this result demonstrated that xenografts from FastPDTX

224 faithfully preserved the primary tumors' genetic characteristics. Above mentioned results indicated

225 that the xenografts from FastPDTX closely resemble the primary tumors.

\section{Drug efficacy can be tested early using pathology}

227 In traditional PDTX, the drug efficacy is assessed by measuring the tumorgraft's volume change

22821 days after the drug treatment. We noticed that in our PDTX tests (Figure 2D) and many other 229 previous studies $(36,37)$, tumorgrafts' volumes have already displayed significant divergence at 230 day seven when treated with drugs with various efficacy. Thereby, we conjectured that the 231 discrepancies would be more distinct if assessed by a microscopic examination inspecting the 232 cellular morphological and pathological changes, and this examination could be performed at an 233 early time point. To test this assumption, we examined necrotic and proliferative rates of tumor 234 cells in the FastPDTX model seven days after the drug treatment. As expected, the drug-treated 235 tumorgraft contained a substantial portion of necrotic cells (Figure 2E, top part, HE stain) and a 236 significantly reduced amount of proliferative cells (Figure 2E, bottom part, Ki67 stain). These 237 results support our opinion that drug efficacy can be assessed by pathological examinations and 238 can be performed at an early time point.

\section{Drug efficacy can be determined by pathological indices}

240 Based on the results mentioned above, we decided to use tumor cell necrotic and proliferative rates 241 as the drug efficacy assessment indices. We adapted one mouse per model per treatment method

242 in FastPDTX since the reproducibility of this scheme has been demonstrated.(25-27) Because the

243 cellular necrosis and the proliferating inhibition could be induced by other conditions such as 244 previous neoadjuvant chemotherapy or an insufficient blood supply in tumorgrafts we used 245 untreated tumorgrafts as a control to abate those potential disturbing effects. We quantified the 
246 percentage of necrotic and proliferative cells and compared those two indices pairwise in control

247 and treated samples. To determine appropriate thresholds that can correctly translate

248 quantifications of these indices into the clinical responses, 12 therapeutic entities where the results

249 of FastPDTX, PDTX, and clinical responses were all available were examined (Table 1 and

250 Supplemental Table 1). We discovered that to make the efficacy assessment reliable, specimens

251 (both control and tested tumorgrafts) should contain at least 5\% tumor cells, and at least one index, 252 either TCNR or Ki67, should be $>5 \%$. On the premise of these two conditions, the difference of

253 TCNR between the tested sample and control was $>10 \%$ (i.e., tested minus control $>10 \%$ ), or the

254 percentage of Ki67 positive cells of the tested sample over control was $<80 \%$ would indicate a

255 positive drug effect, which could be translated into clinical effective disease control

256 (corresponding RECIST criteria CR, PR or SD). In this 12 regimens trial, the positive predictive

257 value (PPV) of FastPDTX was 91.7\%, and PDTX was 83.3\% (Figure 3A, Table 1). Because the

258 negative predictions could not be tested clinically, we did not calculate the sensitivity or specificity.

259 We also compared the concordance between the predictions from FastPDTX and PDTX over 93

260 therapeutic entities, and the concordant rate between the two methods was 69.9\% (Figure 3A,

261 Supplemental Table 1).

\section{FastPDTX predicts clinical outcomes}

263 Comparing with $30-40 \%$ response rate of first-line drugs and $25 \%-10 \%$ of second-line and 264 subsequent-line drugs in standard therapies, using guidance from PDTX, the drug-response rate 265 can be over $80 \%(6,17,38)$. As a novel method, how well the FastPDTX can guide clinic needs 266 to be fully assessed in the context of PDTX and real clinical outcomes being compared. 
267 In the subsequent study, we examined the PPV of FastPDTX and PDTX for efficacious drugs in

268218 cases, where the clinical responses were accessible. However, direct comparisons between

269 PDTX and FastPDTX were not available. The PPV of FastPDTX was 82.3\% (163/198), and PDTX

270 was $81.8 \%$ (27/33); numbers were very close to each other and the previous other study(16) (Figure

271 3B, Supplemental Table 2,3). The PPV results suggested an equivalent performance of FastPDTX

272 to PDTX in guiding efficacious drug selection.

\section{FastPDTX is more appliable clinically}

274 For real-time clinical drug selecting, a drug test should end in a high percentage of appliable test.

275 To assess the performance of FastPDTX in this aspect, we compared two critical indices of

276 FastPDTX and PDTX. The first index is the percentage of successful engraftment or engraftment

277 rate, which is one decisive factor for whether the technique is clinical utilizable. However, due to

278 the distinct technological divergence between the two models, the engraftment rate was defined

279 differently. In our study, for the PDTX model, the successful engraftment was defined as an

280 outgrowth of P0 tumorgafts (volume reaches $60-80 \mathrm{~mm}^{3}$ ) and at least three generations of

281 successful subsequent propagation(39) (Figure 1A). For FastPDTX, the successful engraftment

282 was designated as the living condition of the tumorgraft and established vesiculations before the

283 drug testing started. In the PDTX cohort, 431 cases (from Jan. 2015 to Apr. 2018) were enrolled,

284 covering nine major types of cancer as well as other malignant tumors, and the overall engraftment

285 rate was 57.1\% (246/431) (Figure 3C, D). For the FastPDTX cohort, 1050 cases were enrolled

286 (from Apr. 2018 to Dec. 2019), spanning the same cancer spectrum as the PDTX with a 99.9\%

287 overall engraftment rate (1049/1050) (Figure 3C, D). The second index is the percentage of

288 appliable tests, which we defined as the percentage of tests where the test results were employed

289 successfully for patients. This index reflects the real benefit of FastPDTX under clinical conditions. 
290 For the PDTX group, because of the extended testing period, many patients could not wait until

291 the test finished; therefore, the percentage of appliable tests was low as 16.7\% (72/431), while that

292 of the FastPDTX group was 94.8\% (995/1050) (Figure 3E, F).

\section{FastPDTX allows personalized medicine for tumors hard to manage}

294 As a consequence of the short testing cycle and high ratio of appliable tests (Figure 1A, 3F),

295 FastPDTX is particularly useful for patients with refractory malignant tumor at advanced stage,

296 and in circumstances where classical PDTX has difficulty to be applied. We present three typical

297 cases illustrating how those patients can be benefited from FastPDTX.

298 Case 1(hard-to-grow tumor at advanced stage):

299 A 32-year-old triple-negative breast cancer patient who underwent several rounds of therapies,

300 including surgery, radiotherapy, and several lines of chemotherapy, has developed lung and kidney

301 multiple metastases. Before the testing, the patient already tried epirubicin, paclitaxel liposome,

302 nimotuzumab, docetaxel, and capecitabine. Nine agents were screened with FastPDTX, and

303 gemcitabine was recommended based on the Tumor Cell Necrosis Rate (TCNR) and Ki-67 protein

304 positive staining (Ki67+) results. After 8 cycles of gemcitabine treatment, specific breast cancer

305 markers showed a significant drop: CA153 from 42.21U/L to 30.39U/L, CA125 from 38.26 U/L

306 to $11.06 \mathrm{U} / \mathrm{L}$, and Cyfra21-1 from $16 \mathrm{ng} / \mathrm{ml}$ to $3.81 \mathrm{ng} / \mathrm{ml}$. CT scan showed that the lung and kidney

307 metastases were reduced significantly. The patient gained Partial Response (PR) for eight months

308 (Figure 4A-E).

309 Case 2 (highly advanced malignant tumor):

310 A 49-year-old male with 40 years of hepatitis B positive history was diagnosed with hepatocellular

311 carcinoma (HCC) that recurred in the liver, and he developed multiple metastases in both lungs 
312 three years after the initial diagnosis. The patient has undergone surgery, transarterial

313 chemoembolization (TACE), several lines of conventional treatments, and target therapy

314 (sorafenib, 1 year). Because of the highly advanced tumor, the patient lost the chance for another

315 surgery and was resistant to standard treatments. Six therapeutic entities were tested with

316 FastPDTX, and cabozantinib was recommended. After two cycles of treatment, Alpha-Fetoprotein

317 (AFP) dropped from $1285 \mathrm{ng} / \mathrm{ml}$ to $135 \mathrm{ng} / \mathrm{ml}$, and Protein Induced by Vitamin $\mathrm{K}$

318 Absence/antagonist-II (PIVKA) decreased from 97691mAU/ml to 4686mAU/ml. CT scan showed

319 lung and liver metastasized lesions were reduced significantly. The patient gained PR (Figure 4F-

$320 \mathrm{~J}$ ) and gained the opportunity for debulking surgery.

321 Case 3 (refractory malignant tumor at advanced stage):

322 A 63-year-old male diagnosed with pancreatic duct adenocarcinoma (PDAC) and developed liver

323 metastasis at stage IV. Screening results of FastPDTX showed that fluorouracil, albumin paclitaxel,

324 and oxaliplatin were effective, and Irinotecan was moderately effective. Four weeks after distal

325 pancreatectomy, five cycles of subtraction angiography guided arterial infusion chemotherapies

326 were performed with the combination of FastPDTX guided drugs. The patient's CA-199 dropped

327 from $1000 \mathrm{U} / \mathrm{ml}$ to a normal level at the third cycle of chemotherapy. After five cycles of

328 chemotherapies, the CT scan showed that pancreatic and liver metastatic lesions had disappeared.

329 The clinical response was nearly CR (Figure 4K-O).

\section{FastPDTX matches real clinical practice and increases cost-effectiveness}

331 We reviewed the modeling conditions of FastPDTX and classical PDTX comprehensively. When

332 comparing those conditions to real clinical procedures, we discovered that the modeling conditions

333 of FastPDTX more closely matched the real clinical procedures (Table 2). Moreover, when the

334 costs of FastPDTX and PDTX were compared (China market price), the cost of FastPDTX is only 
335 about 15\% of PDTX, and the cost-effectiveness ratio is almost 40 times higher than PDTX. These

336 results strongly support the clinical utilizability of FastPDTX.

\section{Discussion}

338 Increasing amounts of evidence have shown that PDTX can faithfully preserve the characteristics

339 of original tumors and predict patient response with high accuracy $(19,20,40)$. So far, principal

340 applications of PDTX are researches and preclinical drug screening(26). PDTX has also proven a

341 valuable tool in customized therapy for refractory tumors or rare tumors that clinical guidelines

342 for standard care are usually limited $(15,21,41)$. However, procedures for PDTX modeling are

343 complicated and time-consuming, resulting in low success rates and high costs. Those

344 disadvantages seriously compromise the outstanding value of PDTX for efficacious drug selection

345 in the clinical setting $(14,16,23)$.

346 In this study, we tried to overcome those disadvantages by technical improvements. The pivotal

347 technical alteration is the use of pathological inspections in FastPDTX to replace the measurement

348 of tumor volume in PDTX, which results in two advantages. The first advantage is that the

349 engraftment rate barrier is circumvented. In the traditional PDTX, successful engraftment requires

350 tumorgrafts to be sequentially propagated, and this prerequisite often brings a substandard

351 engraftment rate. In general, the engraftment rate depends on multiple variables, including mouse

352 strains, types of tumors, anatomical locations for engrafting, and many others, which brought

353 significant variances of engraftment rate across different studies (42). In most cases, the rate cannot

354 meet the generally accepted standard of $60 \sim 70 \%$ (14) for personalized medicine. Due to the fast

355 pathological analysis, the FastPDTX modeling simply needs the vital condition of tumorgrafts

356 with vesicular connections for effective drug delivery and does not demand the growth and

357 propagation of tumorgrafts. This alteration substantially improves the portion of successful tests 
358 (Fig. 3D). Implanting tumors in the tissues where blood supply is abundant also helps improve 359 engraftment rates and was reported previously(43). In the FastPDTX protocol development, we 360 used the gluteal region as the primary implanting site and did observe optimized growth of 361 tumorgrafts (Fig. 1B, C). This implanting site alteration is another contributor to the increased 362 success rate.

363 The second advantage is reducing the entire testing cycle from 3-6 months to less than three weeks

364 (Fig. 1A). Comparing with measuring tumor volumes in PDTX, which needs repetitive 365 proliferation of tumorgrafts and thereby causes the extended testing period, the pathological 366 inspections in FastPDTX require just a short growth time for pharmacological effects to take place.

367 This timing reduction produces two significant clinical impacts. The first impact is the 368 considerably improved applicability of FastPDTX (Fig. 3D), which furtherly was translated into 369 the substantially increased number of clinically benefited patients (Fig. 3F). Second, the spectrum 370 of appliable cancer types is broadened. The traditional PDTX modeling demands significant 371 amounts of resources from the host for enough growth of tumorgraft. In particular, for hormone372 driven cancer like breast cancer, the dominant type of female cancers, special requirements like 373 long-term and adequate supplies of hormones are difficult for the mouse host to provide, resulting 374 in a poor engraftment rate in PDTX modeling. In contrast, such a requirement was nonessential in 375 FastPDTX modeling and resulted in superior applicability in the clinical setting. Additionally, 376 indolent tumors in mice, such as neuroendocrine tumors, are also suitable for FastPDTX.

377 Comprehensive pathological indices are used in FastPDTX, including Ki67, TCNR, and 378 percentage of tumor cells. Several factors were considered before those indices were chosen. Ki67 379 proliferative index is used commonly in clinical practice, especially for tumors with high 380 proliferative activity(44-46). In breast cancer, Ki67 has already been listed in the National 
381 Comprehensive Cancer Network (NCCN) guidelines as an essential basis for evaluating prognosis

382 and malignancy(47). Also, TCNR has been used for evaluating tumor cell necrosis caused by

383 therapeutical agents in tumors such as osteosarcoma $(48,49)$. The proportion of tumor cells in the

384 graft is considered to minimize the variances caused by the tumor's heterogeneity. Indeed,

385 pathological examination for efficacy evaluation in PDTX has been implicated in a previous

386 study(50). However, the application of this method as a standard approach has never been reported.

387 To validate the effectiveness of this new pathological approach, we performed an extensive

388 comparison among FastPDTX, PDTX, and clinical outcomes covering >200 clinical cases. The

389 results demonstrated that using this new pathological approach, FastPDTX has equivalent

390 predictive power to PDTX regarding the efficacy evaluation (Fig. 3A, B). Therefore, the validity

391 of this method is proven.

392 Our results showed a high degree of pathological and genetic consistency between grafts and

393 primary tumors (Figure 2), indicating the high fidelity of FastPDTX. During the FastPDTX

394 modeling, the tested graft is at the initial stage, and the test period is relatively short. These two

395 conditions have two implications: First, the elimination of graft passaging can prevent makeup-

396 alterations of the whole graft. After a serial propagation, components of tumorgrafts such as stroma

397 cells will eventually be replaced by murine type, and this change may generate unpredictable

398 outcomes for the efficacy test. Multiple studies have shown various alterations in serially passed

399 PDTX(51), but those alterations were barely found in primary tumorgrafts. Second,

400 microenvironmental-change-induced epigenetic modifications and other genetic changes will be

401 minimized. It has already been known that epigenetic modifications may be responsible for

402 alterations in drug susceptibility (52), and consequently could produce biased test results in animal

403 models. The more frequently grafts are passed and the longer the test period is, the higher the 
404 possibility of epigenetic and genetic alterations and the greater the chance of biased test results.

405 The tested tumorgrafts in FastPDTX are primary tissues with minimum growing time and no 406 passage, which contributes to the reliability of tests.

407 Precision medicine is the future of cancer treatment and, ideally, should be available to the majority 408 of cancer patients. By exclusion of labor-intensive and time-consuming serial passage of 409 tumorgrafts, not only the testing time was significantly shortened in FastPDTX, but the testing 410 cost was also reduced substantially to a feasible range for most families (Table 2).

411 Significant improvements have been made in the FastPDTX system comparing to PDTX. We 412 illustrated three typical cases showing the advantage of FastPDTX in treating the hard-to-grow 413 tumor (case 1, breast cancer), the highly advanced tumor (case 2, hepatocellular carcinoma), and

414 the intractable tumor (pancreatic cancer). Meanwhile, additional issues in which FastPDTX need 415 be improved further, such as cancer type-specific optimized criteria for efficacy evaluating, a 416 pathological categorizing system that can be translated into RECIST equivalent, and multi-omics 417 combined analysis. Regarding the clinical setting, other issues like sample delivery and animal 418 facilities were not addressed in this study and need to be explored in the future. As for clinical 419 decision making, FastPDTX reduces the testing cycle to an acceptable duration (2-3 weeks, 420 comparable to the genetic testing cycle for targeted therapy) and yields over $90 \%$ appliable test 421 while still keeps the same accuracy as PDTX. Therefore, FastPDTX has great potential for real422 time clinical personalized drug selection in combination immunotherapy and traditional standard 423 cancer care.

\section{Acknowledgments:}

425 We are grateful to all the patients, oncologists, pathologist, and laboratory members who 426 participated in this study. 
427 Oncologist and pathologist team: Yuan Cheng, Guang-hai Dai, Bai-yong Shen, Jie-er Ying, Yi Ba, 428 Xin-bo Wang, Ye Xu, Lin Zhou, Ke-feng Ding, Yan-ru Qin, Shu-jun Yang, Wen-xian Guan, Feng 429 Zhang, Xiang-cheng Li, Xiao-feng Wu, Hao Wang, Qaing Li, Xin-bo Wang, Ke Wang, Ye Fan, 430 Yong Zhou, Su-jia Wu, Jian-jie Qin, Wei You, Zhong-ming Tan, Meng Wang, Chuan-yong Zhang,

431 Xiao-Feng Qian, Xin-zheng Dai, Jian Gu, Li-yong Pu, Xiao-xin-Mu, Sheng Han, Chen Wu, Hao 432 Lu, Jian-an Bo, Jun-ming Li, Xiao-zhou Liu, Dong-hua Li, Ping Wang, Jin-dao Wu, Feng Cheng, 433 Chuang-ye Ni, Jian-hua Rao, Zeng-cai Li, Yong-xiang Xia, Chen-yu Jiao, Sen Lu, Chang-xian Li, 434 Ai-hua Yao, Rong-xi Shen, Si-zhen Wang, Hao-ming Zhou, Feng Wang, Xiao-feng Lu, Hao Wang, 435 Sheng-Xian Fan, Long Zhang, Chao Yang, Rong Liu, Zhi-ming Zhao, Jie Gao, Shun-chang Jiao, 436 Guang-hai Dai, Xiao-hui Niu, Yuan-guang Meng, Yan-tao Tian, Qing Zhang, Huan-min Wang, 437 Ling-wei Zhang

438 PDTX laboratory and supporting team: Yan-peng Hao, Guo-feng He, Dan-dan Li, Yan Wang, Yu439 wei Yan, Qi Kong, Tao-zhi Wang, Lin Li, Hong-liang Yan, Chun-xia Li, Jian Cheng, Pei Tao, 440 Jian-bang Xie, Qi Zhang, Xiao-yang Qian, Yu-ting Gao, Liang-liang Chen, Lan Qiu

441 Funding:

442 This work was fully supported by Nanjing Personal Oncology Biotechnology Co. Ltd

\section{Disclosure:}

444 The authors have declared no conflicts of interest.

445 Author contributions: HZ, QW, and YZ developed the main idea and wrote the manuscript; HS 446 participated the discussion and drew figures; $\mathrm{ZW}$ and his team performed the experiments. PY, 447 DF, and YW participated the experimental design and provided samples. YZ provided funding. 
451 1. Gondhowiardjo SA, Handoko, Jayalie VF, Apriantoni R, Barata AR, Senoaji F, et al. Tackling Resistance 452 to Cancer Immunotherapy: What Do We Know? Molecules. 2020;25(18).

453 2. Haslam A, Prasad V. Estimation of the Percentage of US Patients With Cancer Who Are Eligible for and 454 Respond to Checkpoint Inhibitor Immunotherapy Drugs. JAMA Netw Open. 2019;2(5):e192535.

455 3. Bagchi S, Yuan R, Engleman EG. Immune Checkpoint Inhibitors for the Treatment of Cancer: Clinical 456 Impact and Mechanisms of Response and Resistance. Annu Rev Pathol. 2021;16:223 -49.

457 4. Ribas A, Wolchok JD. Cancer immunotherapy using checkpoint blockade. Science. 458 2018;359(6382):1350-5.

459 5. Meric-Bernstam F, Larkin J, Tabernero J, Bonini C. Enhancing anti-tumour efficacy with 460 immunotherapy combinations. Lancet. 2021;397(10278):1010-22.

461 6. Izumchenko E, Meir J, Bedi A, Wysocki PT, Hoque MO, Sidransky D. Patient-derived xenografts as tools 462 in pharmaceutical development. Clin Pharmacol Ther. 2016;99(6):612-21.

463 7. Lominska C, Estes CF, Neupane PC, Shnayder Y, TenNapel MJ, O'Neil MF. CASTLE Thyroid Tumor: A 464 Case Report and Literature Review. Front Oncol. 2017;7:207.

465 8. Aparicio T, Zaanan A, Mary F, Afchain P, Manfredi S, Evans TR. Small Bowel Adenocarcinoma. 466 Gastroenterol Clin North Am. 2016;45(3):447-57.

467 9. Ferrari S, Serra M. An update on chemotherapy for osteosarcoma. Expert Opin Pharmacother. 468 2015;16(18):2727-36.

469 10. Lokesh KN, Anand A, Lakshmaiah KC, Babu KG, Lokanatha D, Jacob LA, et al. Clinical profile and 470 treatment outcomes of metastatic neuroendocrine carcinoma: A single institution experience. South Asian J 471 Cancer. 2018;7(3):207-9.

472 11. Johnson JI, Decker S, Zaharevitz D, Rubinstein LV, Venditti JM, Schepartz S, et al. Relationships between 473 drug activity in NCl preclinical in vitro and in vivo models and early clinical trials. Br J Cancer. 2001;84(10):142447431.

475 12. Day CP, Merlino G, Van Dyke T. Preclinical mouse cancer models: a maze of opportunities and 476 challenges. Cell. 2015;163(1):39-53.

477 13. Ben-David U, Ha G, Tseng YY, Greenwald NF, Oh C, Shih J, et al. Patient-derived xenografts undergo 478 mouse-specific tumor evolution. Nat Genet. 2017;49(11):1567-75.

479 14. Hidalgo M, Amant F, Biankin AV, Budinska E, Byrne AT, Caldas C, et al. Patient-derived xenograft 480 models: an emerging platform for translational cancer research. Cancer Discov. 2014;4(9):998-1013.

481 15. Krepler C, Sproesser K, Brafford P, Beqiri M, Garman B, Xiao M, et al. A Comprehensive Patient-Derived 482 Xenograft Collection Representing the Heterogeneity of Melanoma. Cell Rep. 2017;21(7):1953-67.

483 16. Izumchenko E, Paz K, Ciznadija D, Sloma I, Katz A, Vasquez-Dunddel D, et al. Patient-derived 484 xenografts effectively capture responses to oncology therapy in a heterogeneous cohort of patients with solid 485 tumors. Ann Oncol. 2017;28(10):2595-605.

486 17. Yang W, Fan WS, Ye MX, Li Z, Gu CL, Zhu YP, et al. Establishment of the PDTX model of gynecological 487 tumors. Am J Transl Res. 2019;11(6):3779-89. 
18. Okada S, Vaeteewoottacharn K, Kariya R. Establishment of a Patient-Derived Tumor Xenograft Model and Application for Precision Cancer Medicine. Chem Pharm Bull (Tokyo). 2018;66(3):225-30.

490 19. Tentler JJ, Tan AC, Weekes CD, Jimeno A, Leong S, Pitts TM, et al. Patient-derived tumour xenografts 491 as models for oncology drug development. Nat Rev Clin Oncol. 2012;9(6):338-50.

492 20. Jung J, Seol HS, Chang S. The Generation and Application of Patient-Derived Xenograft Model for 493 Cancer Research. Cancer Res Treat. 2018;50(1):1-10.

494 21. Harris AL, Joseph RW, Copland JA. Patient-derived tumor xenograft models for melanoma drug 495 discovery. Expert Opin Drug Discov. 2016;11(9):895-906.

496 22. Malaney P, Nicosia SV, Dave V. One mouse, one patient paradigm: New avatars of personalized cancer 497 therapy. Cancer Lett. 2014;344(1):1-12.

498 23. Aparicio S, Hidalgo M, Kung AL. Examining the utility of patient-derived xenograft mouse models. Nat 499 Rev Cancer. 2015;15(5):311-6.

500 24. Gock M, Kuhn F, Mullins CS, Krohn M, Prall F, Klar E, et al. Tumor Take Rate Optimization for Colorectal 501 Carcinoma Patient-Derived Xenograft Models. Biomed Res Int. 2016;2016:1715053.

502 25. Einarsdottir BO, Karlsson J, Soderberg EMV, Lindberg MF, Funck-Brentano E, Jespersen H, et al. A 503 patient-derived xenograft pre-clinical trial reveals treatment responses and a resistance mechanism to 504 karonudib in metastatic melanoma. Cell Death Dis. 2018;9(8):810.

505 26. Gao H, Korn JM, Ferretti S, Monahan JE, Wang Y, Singh M, et al. High-throughput screening using 506 patient-derived tumor xenografts to predict clinical trial drug response. Nat Med. 2015;21(11):1318-25.

507 27. Ny L, Rizzo LY, Belgrano V, Karlsson J, Jespersen H, Carstam L, et al. Supporting clinical decision making 508 in advanced melanoma by preclinical testing in personalized immune-humanized xenograft mouse models. 509 Ann Oncol. 2020;31(2):266-73.

510 28. Robinson DR, Wu YM, Lonigro RJ, Vats P, Cobain E, Everett J, et al. Integrative clinical genomics of 511 metastatic cancer. Nature. 2017;548(7667):297-303.

512 29. Li H, Durbin R. Fast and accurate short read alignment with Burrows - Wheeler transform. Bioinformatics. 513 2009;25(14):1754-60.

514 30. Etherington GJ, Ramirez-Gonzalez RH, MacLean D. bio-samtools 2: a package for analysis and 515 visualization of sequence and alignment data with SAMtools in Ruby. Bioinformatics. 2015;31(15):2565-7.

516 31. do Valle IF, Giampieri E, Simonetti G, Padella A, Manfrini M, Ferrari A, et al. Optimized pipeline of 517 MuTect and GATK tools to improve the detection of somatic single nucleotide polymorphisms in whole-exome 518 sequencing data. BMC Bioinformatics. 2016;17(Suppl 12):341.

519 32. Saunders CT, Wong WS, Swamy S, Becq J, Murray LJ, Cheetham RK. Strelka: accurate somatic small520 variant calling from sequenced tumor-normal sample pairs. Bioinformatics. 2012;28(14):1811-7.

521 33. Qidwai T. Exploration of copy number variation in genes related to anti-malarial drug resistance in 522 Plasmodium falciparum. Gene. 2020;736:144414.

523 34. Costa GL, Amaral LC, Fontes CJF, Carvalho LH, de Brito CFA, de Sousa TN. Assessment of copy number 524 variation in genes related to drug resistance in Plasmodium vivax and Plasmodium falciparum isolates from 525 the Brazilian Amazon and a systematic review of the literature. Malar J. 2017;16(1):152. 
35. de Man FM, Goey AKL, van Schaik RHN, Mathijssen RHJ, Bins S. Individualization of Irinotecan Treatment: A Review of Pharmacokinetics, Pharmacodynamics, and Pharmacogenetics. Clin Pharmacokinet. 528 2018:57(10):1229-54.

529 36. Villarroel MC, Rajeshkumar NV, Garrido-Laguna I, De Jesus-Acosta A, Jones S, Maitra A, et al. 530 Personalizing cancer treatment in the age of global genomic analyses: PALB2 gene mutations and the response 531 to DNA damaging agents in pancreatic cancer. Mol Cancer Ther. 2011;10(1):3-8.

532 37. Morelli MP, Calvo E, Ordonez E, Wick MJ, Viqueira BR, Lopez-Casas PP, et al. Prioritizing phase I 533 treatment options through preclinical testing on personalized tumorgraft. J Clin Oncol. 2012;30(4):e45-8.

534 38. Schiff D. Benefit with adjuvant chemotherapy in anaplastic astrocytoma. Lancet. 535 2017;390(10103):1625-6.

536 39. Chen C, Lin W, Huang Y, Chen X, Wang H, Teng L. The Essential Factors of Establishing Patient-derived 537 Tumor Model. J Cancer. 2021;12(1):28-37.

538 40. Prioritizing Phase I Treatment Options Through Preclinical Testing on Personalized Tumorgraft. 539 JOURNAL OF CLINICAL ONCOLOGY. 2012.

540 41. Schatton T, Murphy GF, Frank NY, Yamaura K, Waaga-Gasser AM, Gasser M, et al. Identification of 541 cells initiating human melanomas. Nature. 2008;451(7176):345-9.

542 42. Byrne AT, Alferez DG, Amant F, Annibali D, Arribas J, Biankin AV, et al. Interrogating open issues in 543 cancer precision medicine with patient-derived xenografts. Nat Rev Cancer. 2017;17(4):254-68.

544 43. Read M, Liu D, Duong CP, Cullinane C, Murray WK, Fennell CM, et al. Intramuscular Transplantation 545 Improves Engraftment Rates for Esophageal Patient-Derived Tumor Xenografts. Ann Surg Oncol. 546 2016;23(1):305-11.

547 44. Sorbye H, Welin S, Langer SW, Vestermark LW, Holt N, Osterlund P, et al. Predictive and prognostic 548 factors for treatment and survival in 305 patients with advanced gastrointestinal neuroendocrine carcinoma 549 (WHO G3): the NORDIC NEC study. Ann Oncol. 2013;24(1):152-60.

550 45. Ma X, Wu Y, Zhang T, Song H, Jv H, Guo W, et al. Ki67 Proliferation Index as a Histopathological 551 Predictive and Prognostic Parameter of Oral Mucosal Melanoma in Patients without Distant Metastases. J 552 Cancer. 2017;8(18):3828-37.

553 46. Yerushalmi R, Woods R, Ravdin PM, Hayes MM, Gelmon KA. Ki67 in breast cancer: prognostic and 554 predictive potential. Lancet Oncol. 2010;11(2):174-83.

555 47. Gradishar WJ, Anderson BO, Abraham J, Aft R, Agnese D, Allison KH, et al. Breast Cancer, Version 556 3.2020, NCCN Clinical Practice Guidelines in Oncology. J Natl Compr Canc Netw. 2020;18(4):452-78.

557 48. Ji F, LV R, Zhao T. A correlation analysis between tumor imaging changes and $p$-AKT and HSP70 558 expression in tumor cells after osteosarcoma chemotherapy. Oncol Lett. 2017;14(6):6749-53.

559 49. Xia W, Yan Z, Gao X. Volume fractions of DCE-MRI parameter as early predictor of histologic response 560 in soft tissue sarcoma: A feasibility study. Eur J Radiol. 2017;95:228-35.

561 50. Wang $Y$, Ding $X$, Wang S, Moser CD, Shaleh HM, Mohamed EA, et al. Antitumor effect of FGFR 562 inhibitors on a novel cholangiocarcinoma patient derived xenograft mouse model endogenously expressing 563 an FGFR2-CCDC6 fusion protein. Cancer Lett. 2016;380(1):163-73.

$56451 . \quad$ Shi J, Li Y, Jia R, Fan X. The fidelity of cancer cells in PDX models: Characteristics, mechanism and 565 clinical significance. Int J Cancer. 2020;146(8):2078-88. 
566 52. Nikolaou M, Pavlopoulou A, Georgakilas AG, Kyrodimos E. The challenge of drug resistance in cancer 567 treatment: a current overview. Clin Exp Metastasis. 2018;35(4):309-18.

568

569 


\section{Figure 1. General comparisons between FastPDTX and PDTX}

572 (A) Schematic diagram showing the work flow and time frame of FastPDTX (top) and PDTX

573 (bottom). (B) An example showing distinct growth rates of tumorgrafts using FastPDTX or PDTX

574 protocol. (C) Rapid vascularization in FastPDTX tumorgrafts (showing four samples from

575 different cases); arrows indicate either blood vascular (left) or CD31 positive staining (right, brown

576 stained). (D) Specific growth rate (SGR) of various tumorgrafts under FastPDTX or PDTX 577 conditions.

578 Figure 2. Establishment of pathological examination for efficacy assessment

579 (A) Histopathologic inspections (hematoxylin and eosin stain) of 7 pairs of original tumors (Patient) 580 and their corresponding tumorgrafts (FastPDTX). (B) Copy number variations (CNVs) in the 7 581 tumorgrafts measured by $\mathrm{Z}$ score; $\mathrm{Z}$ score $>3$ indicates significant copy number alteration. (C) 582 Single nucleotide variations (SNVs) in tumorgrafts (P0) and original tumors (Pa). Minor allele 583 frequency (MAF) was compared side by side (P0 vs. Pa). Each red circle indicates an allele. Blue 584 straight line shows the ideal situation (no changes between P0 and Pa); red straight line shows the 585 real results. (D) A typical growth curve of tumorgrafts in PDTX protocol. Red arrow indicates 586 diverse tumor volumes at day 7 when treated with different drugs. (E) Distinct pathological 587 patterns in drug treated tumorgrafts. Tumorgraft was examined 7 days after cabozantinib treatment. 588 Red arrow and red dashed line circled area indicate necrotic cells; Blue arrows indicate live cells; 589 Brown color shows Ki67 positive cells.

590 Figure 3. Comprehensive Comparison between FastPDTX and PDTX 
591 (A) Pairwise comparison of positive predictive values for drug efficacy and the concordant rate 592 between FastPDTX and PDTX. (B) Positive predictive values of FastPDTX and PDTX in 593 independent cases. (C) Number of enrolled cases across various type of solid tumor in FastPDTX

594 (blue) or PDTX (green). (D) Engraftment rate and percentage of appliable tests of FastPDTX and 595 PDTX among various type of cancers. (E) Number of clinical benefited patients from FastPDTX 596 or PDTX in this study since 2015. (F) The percentage of applicable test of FastPDTX or PDTX in 597 this study since 2015.

598 Figure 4. Typical cases from FastPDTX

599 (A-E) Triple-negative breast cancer case: (A) testing regimens, agents, and tumor components. (B) 600 histopathology staining (HE) and statistics of TCNR. (C) Ki67 staining (IHC) and statistics. (D) 601 changes of breast cancer specific marker CA153, CA125 and Cyfra21-1. (E) CT scan images 602 before and after treatment. (F-J) Corresponding regimens, statistics, biomarkers, or images from 603 the advanced liver cancer case. (K-O) Corresponding regimens, statistics, biomarker, or images 604 from the advanced pancreatic cancer case. 
606

\begin{tabular}{|c|c|c|c|c|c|c|c|c|c|c|c|}
\hline \multirow{2}{*}{$\begin{array}{l}\text { Case } \\
\text { ID }\end{array}$} & \multirow[b]{2}{*}{ Type } & \multirow[b]{2}{*}{ Regimen } & \multicolumn{4}{|c|}{ FastPDTX } & \multicolumn{2}{|c|}{ PDTX } & \multirow{2}{*}{$\begin{array}{l}\text { Clinical } \\
\text { response }\end{array}$} & \multicolumn{2}{|c|}{ Congruous between } \\
\hline & & & $\begin{array}{c}\text { Tumor cell } \\
(\%)\end{array}$ & TCNR & Ki67 & Prediction & TGI & Prediction & & $\begin{array}{c}\text { Clinical/ } \\
\text { FastPDTX }\end{array}$ & $\begin{array}{c}\text { Clinical/ } \\
\text { PDTX }\end{array}$ \\
\hline \multirow{3}{*}{050106} & \multirow{3}{*}{ OS } & Control & $40 \%$ & $0 \%$ & $60 \%$ & & & & & & \\
\hline & & Docetaxel+Gemcitabine & $10 \%$ & $10 \%$ & $60 \%$ & $P$ & $77.3 \%$ & $\mathrm{P}$ & PR & $\mathrm{Y}$ & $\mathrm{Y}$ \\
\hline & & Etoposide+Ifosfamide & $40 \%$ & $20 \%$ & $70 \%$ & $\mathrm{P}$ & $86.7 \%$ & $\mathrm{P}$ & PR & $\mathrm{Y}$ & $\mathrm{Y}$ \\
\hline \multirow{2}{*}{050125} & \multirow{2}{*}{ PC } & Control & $20 \%$ & $5 \%$ & $50 \%$ & & & & & & \\
\hline & & Gemcitabine+Tegafur & $30 \%$ & $0 \%$ & $10 \%$ & $P$ & $80.5 \%$ & $P$ & CR & $\mathrm{Y}$ & $\mathrm{Y}$ \\
\hline \multirow{2}{*}{080093} & \multirow{2}{*}{$\mathrm{CRC}$} & Control & $70 \%$ & $0 \%$ & $70 \%$ & & & & & & \\
\hline & & Irinotecan+Cetuximab & $10 \%$ & $0 \%$ & $20 \%$ & $\mathrm{P}$ & $76.80 \%$ & $\mathrm{P}$ & SD & $\mathrm{Y}$ & $\mathrm{Y}$ \\
\hline \multirow{2}{*}{101007} & \multirow{2}{*}{ OC } & Control & $20 \%$ & $20 \%$ & $20 \%$ & & & & & & \\
\hline & & Bevacizumab+Paclitaxel+Lobaplatin & $20 \%$ & $30 \%$ & $10 \%$ & $\mathrm{P}$ & $47.30 \%$ & $\mathrm{~N}$ & CR & $\mathrm{Y}$ & $\mathrm{N}$ \\
\hline \multirow{2}{*}{110150} & \multirow[b]{2}{*}{ GC } & Control & $70 \%$ & $10 \%$ & $90 \%$ & & & & & & \\
\hline & & Albumin paclitaxel+Cisplatin & $30 \%$ & $5 \%$ & $40 \%$ & $\mathrm{P}$ & $91.60 \%$ & $\mathrm{P}$ & SD & $\mathrm{Y}$ & $\mathrm{Y}$ \\
\hline \multirow{2}{*}{130111} & \multirow{2}{*}{ PC } & Control & $8 \%$ & $0 \%$ & $70 \%$ & & & & & & \\
\hline & & Gemcitabine & $2 \%$ & $0 \%$ & $<5 \%$ & $\mathrm{P}$ & $86.70 \%$ & $\mathrm{P}$ & SD & $\mathrm{Y}$ & $\mathrm{Y}$ \\
\hline \multirow{2}{*}{200171} & \multirow{2}{*}{ os } & Control & $20 \%$ & $0 \%$ & $5 \%$ & & & & & & \\
\hline & & Docetaxel+Gemcitabine & $1 \%$ & $10 \%$ & $3 \%$ & $\mathrm{P}$ & $61.5 \%$ & $\mathrm{P}$ & SD & $\mathrm{Y}$ & $\mathrm{Y}$ \\
\hline \multirow{2}{*}{212001} & \multirow{2}{*}{ SS } & Control & $60 \%$ & $0 \%$ & $75 \%$ & & & & & & \\
\hline & & lipo-doxorubicin & $95 \%$ & $0 \%$ & $60 \%$ & $\mathrm{P}$ & $68.2 \%$ & $\mathrm{P}$ & SD & $\mathrm{Y}$ & $\mathrm{Y}$ \\
\hline \multirow{5}{*}{240154} & \multirow{5}{*}{ os } & Control & $80 \%$ & $5 \%$ & $50 \%$ & & & & & & \\
\hline & & Apatinib & $70 \%$ & $0 \%$ & $80 \%$ & $\mathrm{~N}$ & $45.0 \%$ & $\mathrm{~N}$ & PD & $\mathrm{Y}$ & $\mathrm{Y}$ \\
\hline & & Gemcitabine+Docetaxel & $80 \%$ & $20 \%$ & $60 \%$ & $\mathrm{P}$ & $97.9 \%$ & $\mathrm{P}$ & SD & $\mathrm{Y}$ & $\mathrm{Y}$ \\
\hline & & Etoposide+Ifosfamide & $30 \%$ & $0 \%$ & $10 \%$ & $\mathrm{P}$ & $96.2 \%$ & $P$ & PD & $\mathrm{N}$ & $\mathrm{N}$ \\
\hline & & \multicolumn{8}{|c|}{ Positive Predictive Value } & $11 / 12$ & $10 / 12$ \\
\hline
\end{tabular}

607 Abbreviations: $\mathrm{OS}=$ osteosarcoma, $\mathrm{PC}=$ pancreatic cancer, $\mathrm{CRC}=$ colorectal cancer, OC=0varian cancer, $\mathrm{GC}=\mathrm{gastric}$ cancer,

608 SS=synovial sarcoma, $\mathrm{HCC}=$ hepatocellular carcinoma. In the prediction column, $\mathrm{P}=$ a positive drug effect that equivalent to $\mathrm{CR}$ or PR

609 or SD; $\mathrm{N}=$ a negative drug effect that equivalent to PD. 


\begin{tabular}{|c|c|c|c|c|}
\hline & & FastPDTX & PDTX & RCT \\
\hline \multirow{4}{*}{$\begin{array}{c}\text { Xenograft } \\
\text { characteristics }\end{array}$} & $\begin{array}{c}\text { Tumor } \\
\text { microenvironment }\end{array}$ & $\begin{array}{l}\text { Original microenvironment } \\
\text { is kept at great extent }\end{array}$ & $\begin{array}{l}\text { Stroma cells may be } \\
\text { replaced by murine type }\end{array}$ & $\begin{array}{l}\text { Original } \\
\text { microenvironment }\end{array}$ \\
\hline & Tumor architecture & $\begin{array}{c}\text { Original stereoscopic } \\
\text { structure }\end{array}$ & $\begin{array}{l}\text { Original stereoscopic } \\
\text { structure (P0) }\end{array}$ & $\begin{array}{c}\text { Original } \\
\text { stereoscopic } \\
\text { structure }\end{array}$ \\
\hline & $\begin{array}{l}\text { Histopathological } \\
\text { consistency }\end{array}$ & Almost full uniform (PO) & $\begin{array}{c}\text { Highly uniform } \\
\text { (decreasing with passages) }\end{array}$ & Original tissue \\
\hline & $\begin{array}{l}\text { Genetic consistency } \\
\text { with original tumor }\end{array}$ & Almost full uniform(P0) & $\begin{array}{l}\text { Highly uniform (decreasing } \\
\text { with passages) }\end{array}$ & Original tissue \\
\hline \multirow{8}{*}{$\begin{array}{l}\text { Drug } \\
\text { administration }\end{array}$} & tumor tissue & $\mathrm{PO}$ & P1-P5 & Primary tumor $(\mathrm{Pa})$ \\
\hline & Tumor size & $8-12 \mathrm{~mm}^{3}$ & $100-150 \mathrm{~mm}^{3}$ & Original size \\
\hline & \multirow{2}{*}{ Administration Route } & Gavage & Gavage & Oral \\
\hline & & Intraperitoneal injection & Intraperitoneal injection & Intravenous (IV) \\
\hline & $\begin{array}{l}\text { Starting point for } \\
\text { patient }\end{array}$ & $15-20$ days (P0) & 80-200 days (P1-P5) & $\begin{array}{c}\text { 20-30 days after } \\
\text { surgery }\end{array}$ \\
\hline & Drug regimen & $\begin{array}{l}\text { Multiple regimens } \\
\text { simultaneously }\end{array}$ & $\begin{array}{l}\text { Multiple regimens } \\
\text { simultaneously }\end{array}$ & One regimen \\
\hline & Drug dose & $\begin{array}{l}\text { Converted from clinical } \\
\text { dosage }\end{array}$ & $\begin{array}{l}\text { Converted from clinical } \\
\text { dosage }\end{array}$ & Clinical guideline \\
\hline & Evaluation criteria & Pathology, TCNR, Ki67 & TGI & biomarkers, CT \\
\hline
\end{tabular}




\begin{tabular}{|c|c|c|c|c|}
\hline \multirow{2}{*}{ Efficacy } & Test sensitivity & Sensitive & Moderately sensitive & Sensitive \\
\cline { 2 - 5 } assessment & Testing cycle & About 20 days & About 120-180 days & - \\
\hline \multirow{2}{*}{ Cost-efficiency } & Average & $\sim 4500$ USD & $\sim 30,000$ USD & - \\
\cline { 2 - 5 } & Price/patient* & & & \\
& Cost-effectiveness & & & \\
& (USD/patient) & & & \\
\hline
\end{tabular}

$612 *$ According to Chinese market price, test of four therapeutic entities. 
Figure 1

A

Efficacy test results

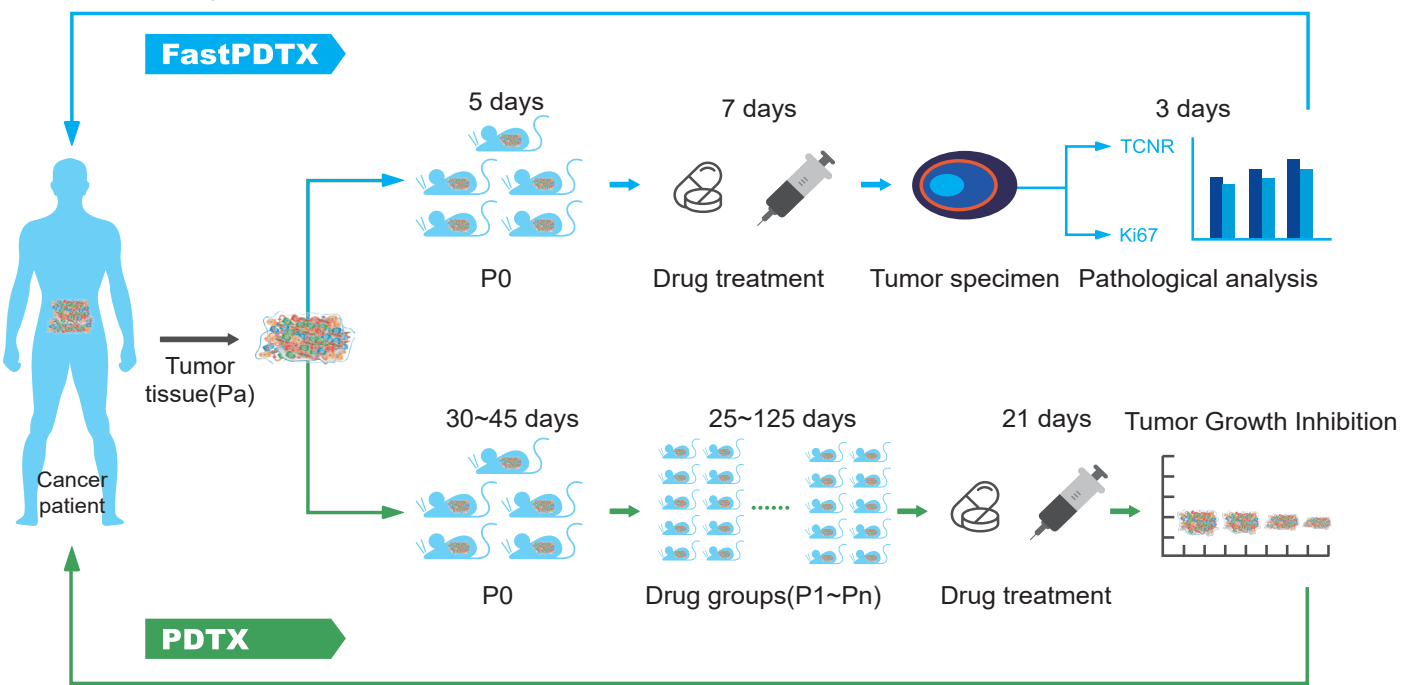

Efficacy test results

FastPDTX

15 20 days

PDTX

80 200 days

B

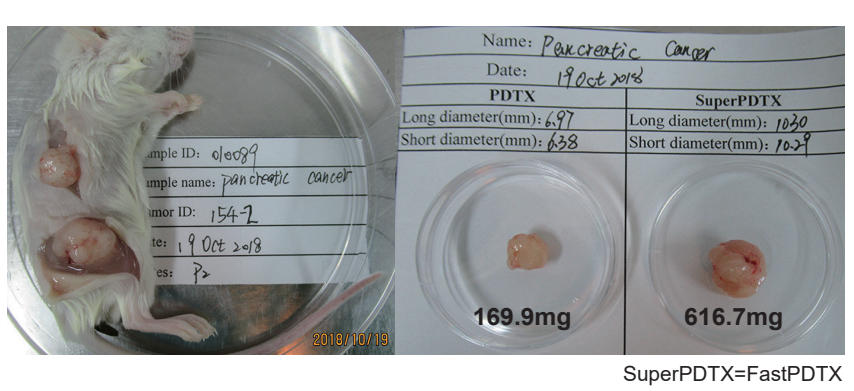

C

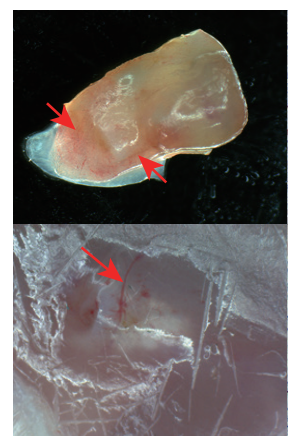

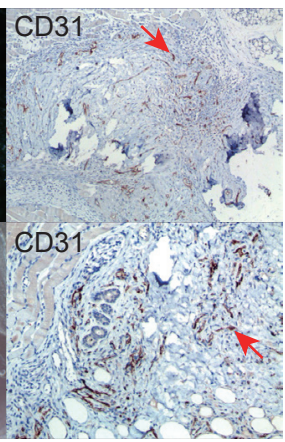

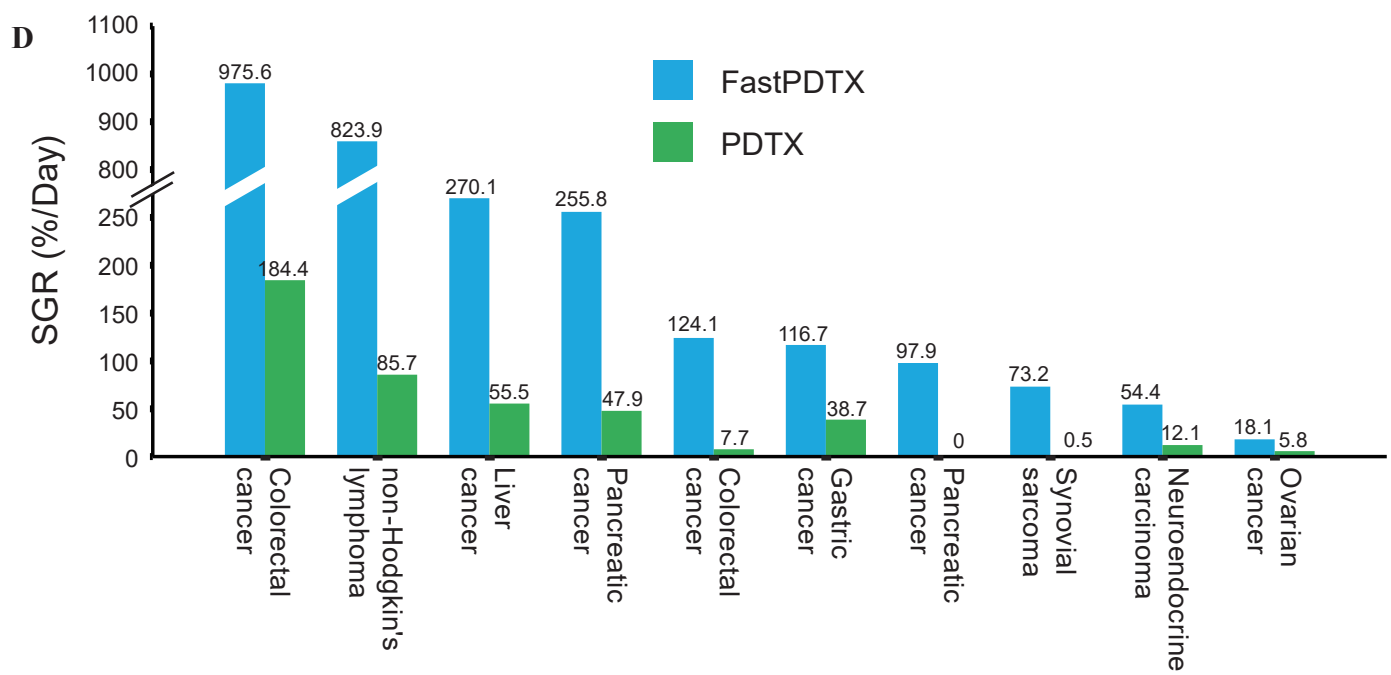


Figure 2

A

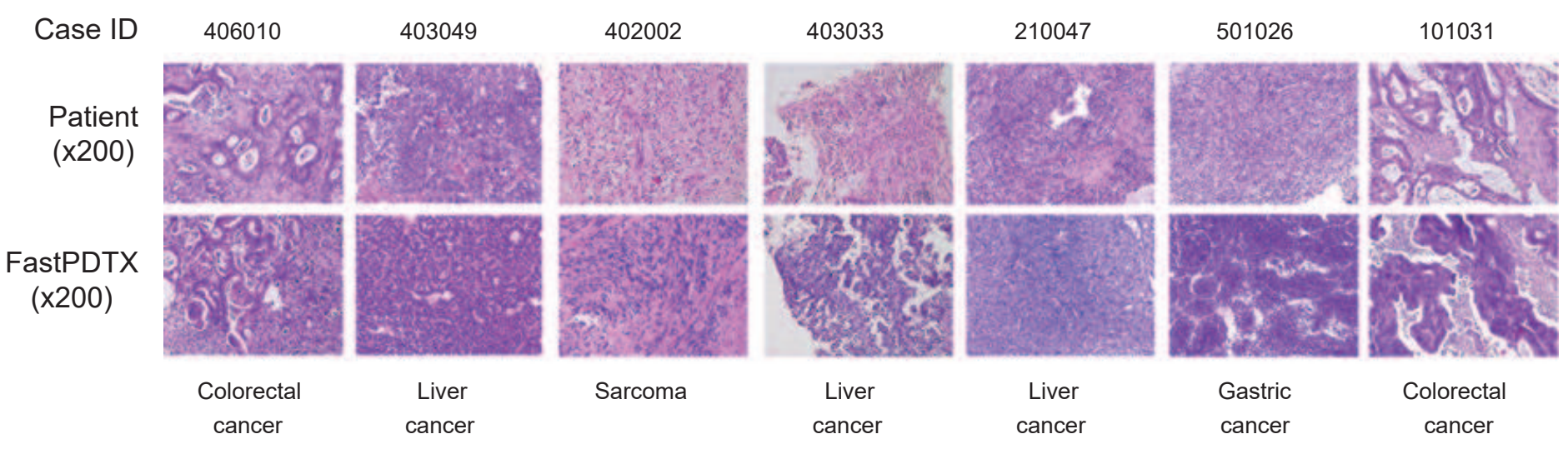

\section{B}
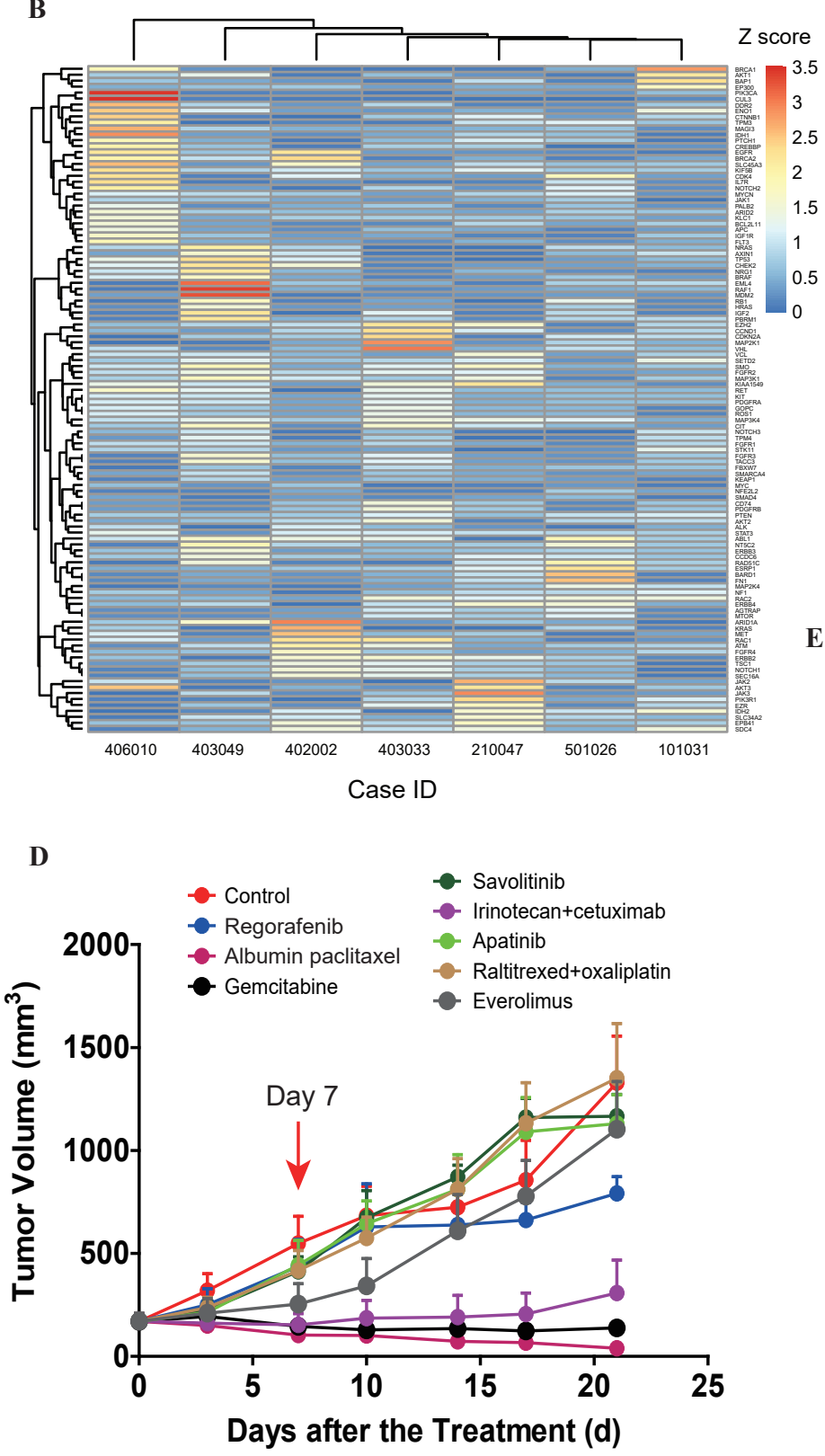

C

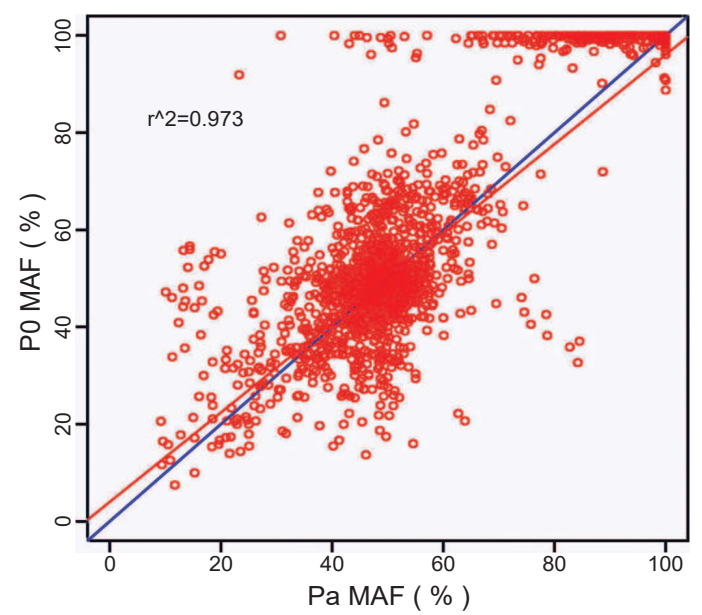

E

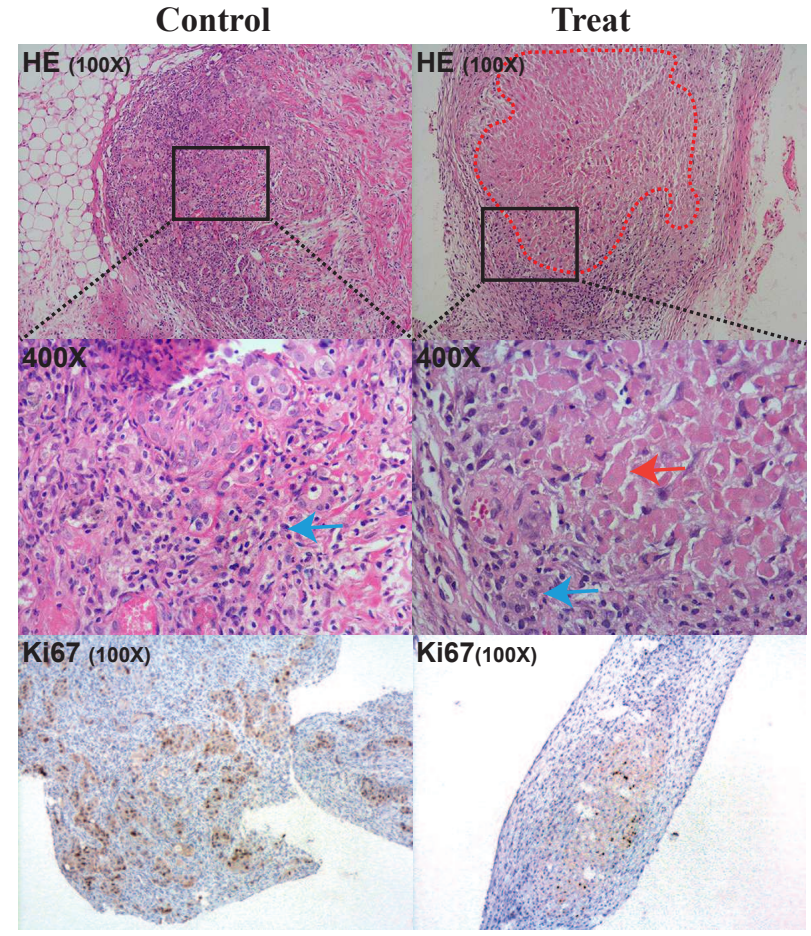


Figure 3
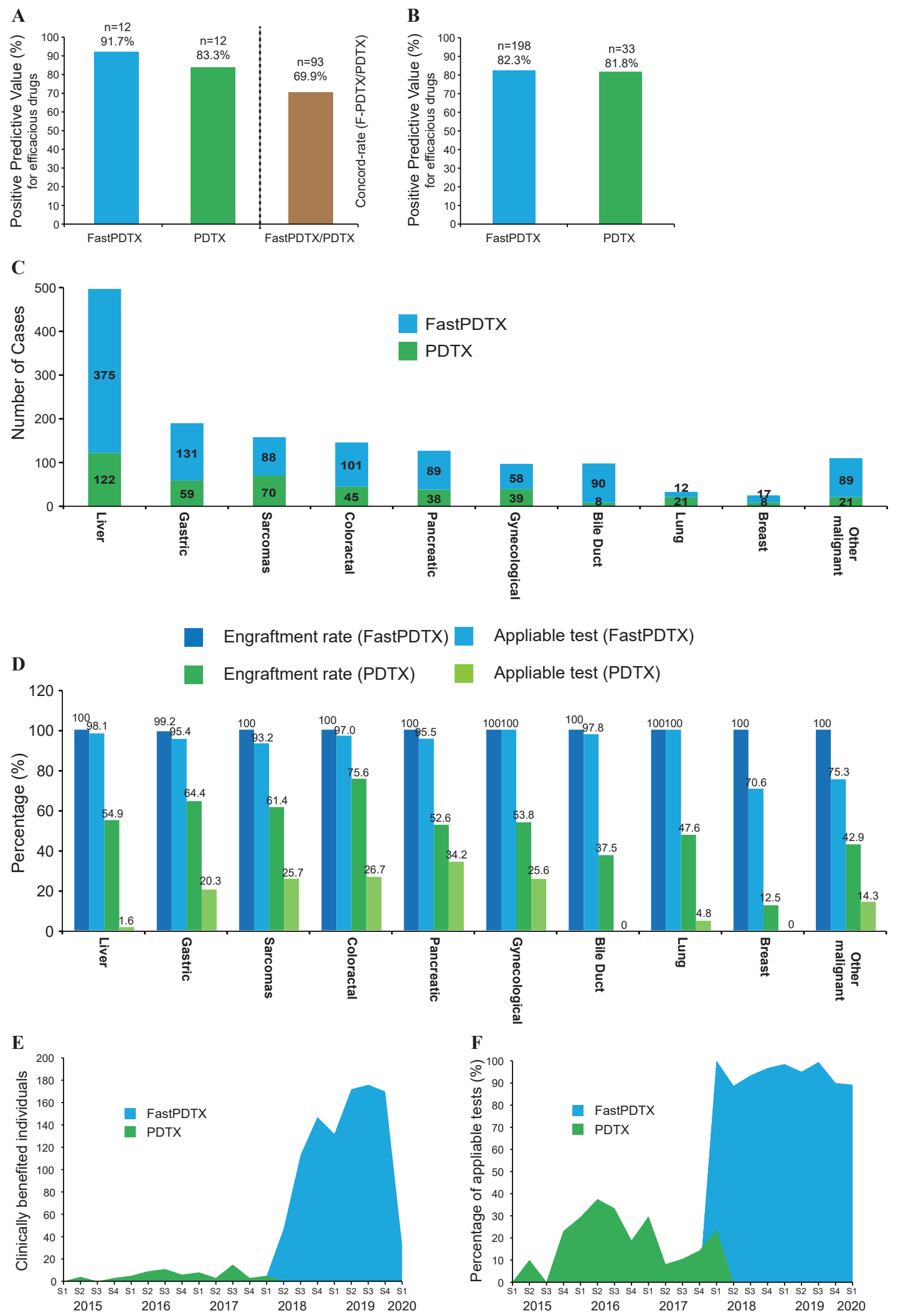
Figure 4

A

\begin{tabular}{ccc}
\hline Regimens & Agents & Tumor component (\%) \\
\hline 1 & Control & 60 \\
2 & Vinorelbine & 50 \\
3 & Cisplatin & 50 \\
4 & Gemcitabine & 50 \\
5 & Nedaplatin & 40
\end{tabular}

HE Albumin paclitaxel

Pemetrexed

Apatinib

Olaparib

Capecitabin

5



7

6
E
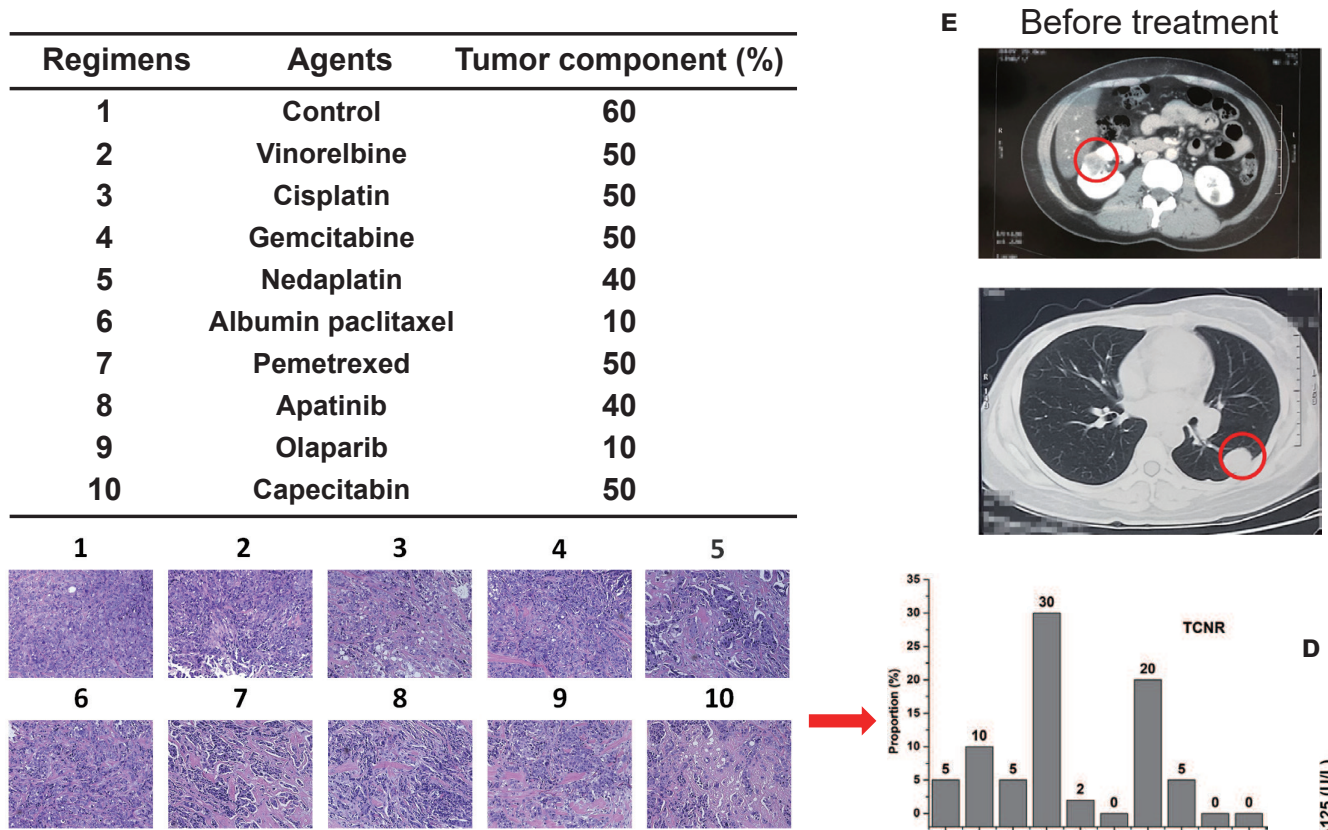

C

Ki-67
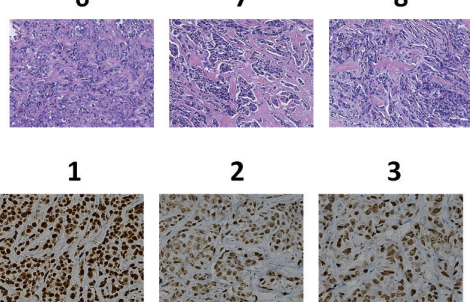

2

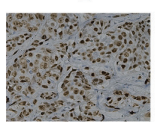

7

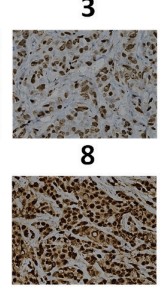

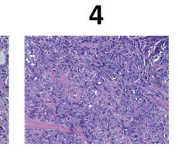

9

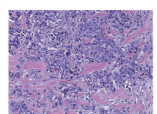

4

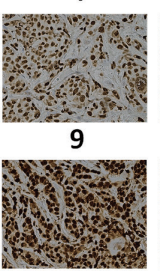

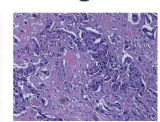

10

5



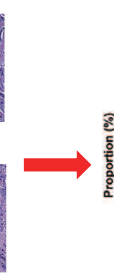
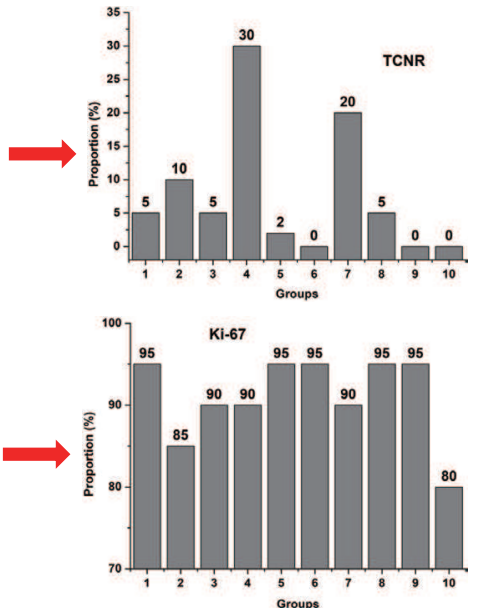
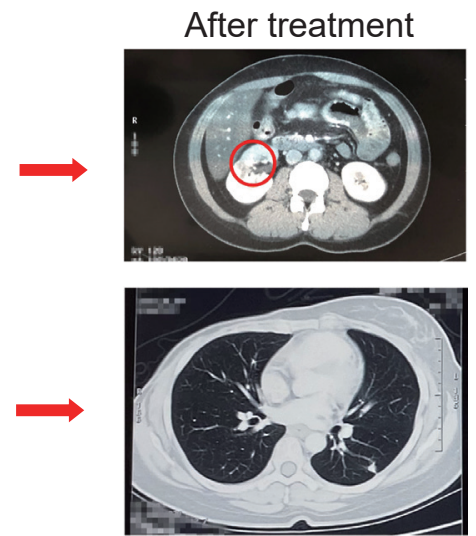

F

\begin{tabular}{ccc}
\hline Regimens & Agents & Tumor component (\%) \\
\hline 1 & Control & 50 \\
2 & Lenvatinib & 20 \\
3 & Lenalidomide & 50 \\
4 & Regorafenib & 50 \\
5 & Nedaplatin & 50 \\
6 & Everolimus+FOLFOX & 60 \\
7 & Cabozantinib & 80 \\
\hline
\end{tabular}
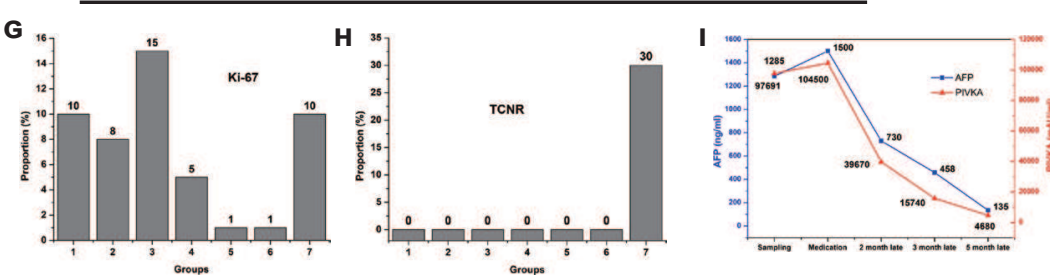

K

\begin{tabular}{ccc}
\hline Regimens & Agents & Tumor component (\%) \\
\hline 1 & Control & 50 \\
2 & Fluorouracil & 20 \\
3 & Gemcitabine & 50 \\
4 & Albumin paclitaxel & 50 \\
5 & Oxaliplatin & 50 \\
6 & Irinotecan & 60 \\
\hline
\end{tabular}
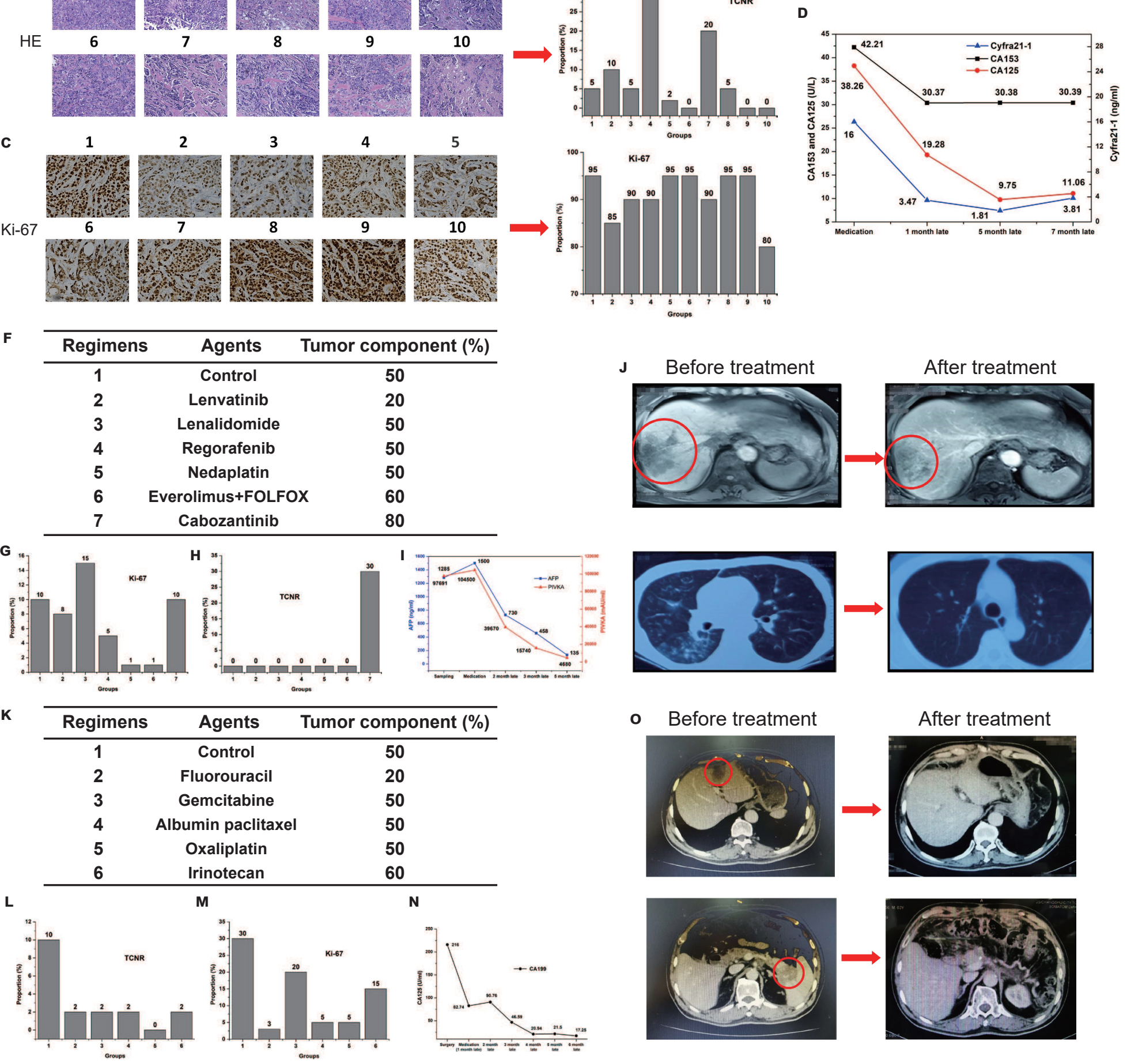

- Before treatment
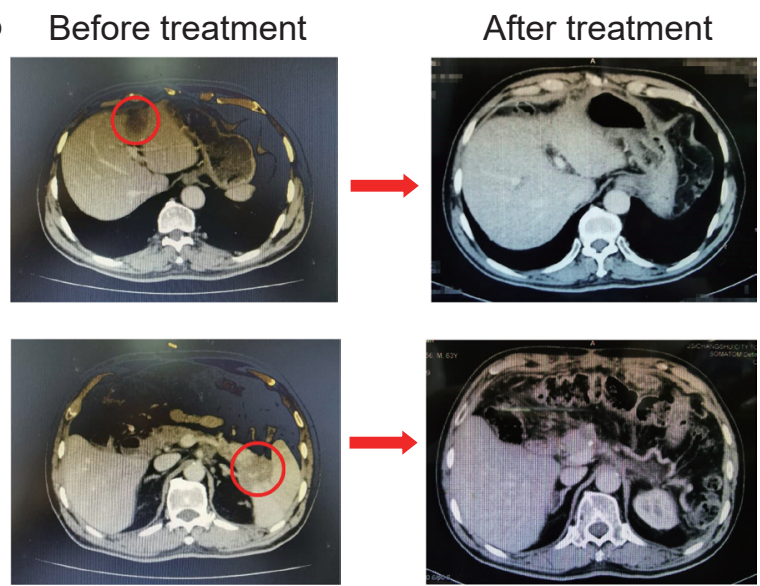


\section{Supplementary Files}

This is a list of supplementary files associated with this preprint. Click to download.

- Supplementaldata.xlsx 